\title{
Overview of Distribution Network Fault Location
}

\author{
Hao Wu, Jie Yang, Qiaomei Wang, Leilei Chen \\ Automation and Information Engineering, Sichuan University of Science \& Engineering, Zigong, China \\ Email: wuhao801212@163.com
}

How to cite this paper: $\mathrm{Wu}, \mathrm{H}$., Yang, J., Wang, Q.M. and Chen, L.L. (2019) Overview of Distribution Network Fault Location. Energy and Power Engineering, 11, 209-219.

https://doi.org/10.4236/epe.2019.115013

Received: April 30, 2019

Accepted: May 27, 2019

Published: May 30, 2019

Copyright $\odot 2019$ by author(s) and Scientific Research Publishing Inc. This work is licensed under the Creative Commons Attribution International License (CC BY 4.0).

http://creativecommons.org/licenses/by/4.0/

(c) (i) Open Access

\begin{abstract}
According to the existing research, the fault section location and fault location of passive distribution network and active distribution network are reviewed. Among them, fault location of passive distribution network mainly introduces fault segment location based on transient state and steady state quantity and fault location based on transient quantity. The active distribution network mainly introduces the fault segment location based on the current amount and the switching capacity based on the distribution network topology. On this basis, the difficulties of fault location in the distribution network at present are analyzed, and the future development is prospected.
\end{abstract}

\section{Keywords}

Passive Distribution Network, Active Distribution Network, Segment Location, Fault Location, Research and Prospect

\section{Introduction}

With the increasing demand for electrical energy in people's daily production and life, the density of distribution networks is also increasing. When the distribution network fails, the accurate fault location of the distribution network is the premise for realizing the rapid and effective removal of the distribution network fault and restoring the power supply. This plays an important role in ensuring the power quality of the user and improving the system reliability [1].

The automatic fault location of distribution network is divided into fault line selection, fault section location and fault location according to the requirements and objectives of fault location. The characteristics of the implementation difficulty are gradually increased. For a long time, scholars at home and abroad (such as: Song Guobing, Zhang Wei, etc.) have carried out a large number of theoretical and experimental studies in these three aspects, among which the re- 
sults in fault line selection are quite abundant, and a large number of industrial products have been applied to the field, but in the fault section positioning and failure Research on ranging still faces many problems, and a lot of theoretical and practical research is needed. Based on the existing research results, this paper comprehensively discusses the fault interval location and fault location from the passive distribution network and the active distribution network, and analyzes the difficulties in various aspects, and gives the basis of suggestions and prospects for future development.

\section{Passive Distribution Network Fault Location}

Most of China's medium voltage distribution network is a small current grounding system. Single-phase ground fault is the main type of fault in the distribution network, accounting for a large proportion of total line faults. Safe and reliable operation has an important impact [2]-[7]. This section mainly discusses the single-phase grounding fault of the small current grounding system in the passive power distribution network. It mainly introduces the fault segment location based on transient state and steady state quantity and the fault location based on transient quantity. The fault segment location based on the transient electrical quantity mainly utilizes the direction measurement information of the electrical characteristics of the current and voltage quantities of each measurement point of the line and the obtained waveform similarity, energy relative entropy or line transient center of gravity frequency and other information. To determine the fault section; the fault section location based on the steady state electrical quantity mainly uses the current and voltage information of each measurement point, the correlation coefficient of the structure is used to realize the fault section positioning; the fault traveling wave distance measurement based on the transient quantity Mainly utilize the traveling wave characteristics generated when the line is faulty, and realize the traveling wave distance measurement of the fault according to the ranging algorithm.

\subsection{Fault Segment Location Based on Transients}

The fault segment location method based on transient components mainly uses fault transient fault component information to construct new physical quantities and obtain relative energy entropy to identify fault segments. In [8], a unified objective function is constructed based on the fundamental zero-sequence current fault direction measure and the transient phase current fault direction measure information, and the objective function is used to find the optimal solution to realize the single-phase ground fault zone of the neutral point ungrounded distribution network. Segment positioning, in the algorithm [8] using the optimization solution thinking, improve the fault tolerance of the algorithm.

In [9], the fault direction parameter $D$ is constructed by multiplying the transient line voltage and the transient zero mode current after the Hilbert transform, and the fault section is judged by comparing the positive and negative 
characteristics of the fault direction parameters uploaded by each detection point. In the case of single-phase ground fault, when the first $D>0$ occurs, the line segment between the detection point and the previous detection point is the fault section; if the fault direction parameter $D<0$ is obtained by all the detection points, the line end is faulty.

In [10], the S-transform of the transient fault component of the transient zero-mode current is firstly performed, and then the fault characteristic frequency is determined according to the energy and the maximum principle, and then the relative entropy of the transient zero-mode current energy of any two adjacent detection points at the characteristic frequency is obtained. In the end, the multi-combination method is used to compare the obtained entropy values to identify the faulty segment; in [11], based on the analysis of the relationship between the upstream and downstream zero-sequence current transient spectrum of the fault point, according to the zero-sequence current of the line on both sides of the fault point The characteristics of different center-of-gravity frequencies are proposed to realize the fault segment location by using the line transient center-of-gravity frequency and the line center-of-gravity frequency amplitude information. In order to improve the accuracy of fault section location, literature [11] adopts k-means clustering algorithm to perform clustering analysis on the transient barycenter frequency of each section, so as to reduce the error of line frequency extraction in the process of fast Fourier transform.

For the characteristics of the transient zero-sequence current similarity at both ends of the healthy segment and the transient zero-sequence current difference between the two ends of the fault segment, the similarity between the transient zero-mode currents on both sides of the segment is used in [12]. Judging the fault zone, but using the transient current similarity relationship location method to locate the blind zone, the literature [13] for the literature [12] positioning blind zone problem, the transient current similarity and polarity relationship between the upstream and downstream of the integrated section, proposed The fault section is identified by the transient zero-mode current correlation coefficient obtained by synthesizing the transient zero-mode current and the power-frequency zero-sequence current correlation coefficient between adjacent detection points of the line. In [14], a fault segment localization method based on zero-sequence current generalized S-transform energy relative entropy is proposed. The fault zone is identified by the relative entropy sum of the energy of any two adjacent detection points.

\subsection{Fault Segment Location Based on Steady State Quantity}

Reference [15] uses the correlation coefficient between the fault phase and the non-fault phase current abrupt waveform to realize the location of the fault section of the line, but it is necessary to ensure the synchronization of the data of each phase, and the adjustment of the correlation coefficient is susceptible to the operation mode of the distribution network. In order to overcome the above 
shortcomings, the literature [16] realizes the single-phase ground fault section location of the distribution network by calculating the correlation coefficient between the phase current sudden change and the phase voltage sudden change derivative of each section of the distribution network line in a specific frequency band. When the correlation coefficient is negative, it can be determined that the segment is before the fault point, and the segment having the negative correlation coefficient farthest from the busbar is the fault segment. When the correlation coefficient is positive, it is determined to be a bus fault. In [17], based on the analysis of the line branch fault at the outlet end of the substation, the branch fault of the non-output line, and the zero-sequence current characteristics before and after the fault point, the zero-sequence current sudden change is proposed for the single-phase ground fault of the resonant grounding distribution network. Identify the line fault section. When the zero-sequence current before and after the arc-suppression coil parameter change is converted to the same voltage condition, the line with the zero-sequence current sudden change amount $\left|\Delta \dot{I}_{i}\right| \neq 0$ is the fault line; When the zero sequence current sudden change of all lines is $\left|\Delta \dot{I}_{i}\right|=0$, it is determined as the bus line fault. In the faulty line, the section with the largest zero-sequence current $\left|\Delta \dot{I}_{i}\right|$ mutation is the fault section.

\subsection{Transient-Based Fault Traveling Wave Ranging}

In [18], the single-phase ground fault charging and discharging transient characteristics of the resonant grounding system are introduced into the distribution network fault location, and the discharge transient frequency range is judged according to the line fault charging transient frequency characteristic, which is integrated in the ranging algorithm. The fault distance is calculated by the characteristics of the line fault charging transient frequency and the fault discharge transient frequency, so as to achieve accurate positioning of the fault point of the distribution network. Compared In [19], the fault signal is analyzed by the combination of frequency domain analysis and time domain analysis, so as to determine the arrival time of the reflected wave head of the opposite side of the fault line, and then use the time difference between the initial wave head and the opposite side bus reflection head to achieve accurate fault location. In [20], the peer equivalent impedance is estimated online, and the fault section method is derived from the single-ended power frequency segment by segment. The fault point position $d$ and the fault distance interval $D$ are initially determined, and the traveling wave propagation law in the interval is used. The method of accurately constructing the fault point precise positioning method, the connection point fault identification criterion and the fault section criterion is to realize the single-ended traveling wave fault location of the hybrid transmission line.

\section{Active Distribution Network Fault Location}

Distributed generation is a small-scale power generation system with a capacity 
of less than $50 \mathrm{MW}$ and is installed on the user side and compatible with the environment. Compared with traditional centralized power generation technology, distributed generation technology is clean, efficient, environmentally friendly, energy diversified, and in line with national sustainable development. Many advantages of strategy [21] [22] [23]. With the continuous maturity of small-scale power generation technologies that utilize new energy sources, a large number of distributed power sources are connected to the distribution network, turning the traditional distribution network from a passive network into an active network. For multi-source networks with complex DG fault characteristics, the selectivity of protection is difficult to satisfy only by relying on single-ended information, which brings new challenges to fault location of distribution networks. This section mainly introduces the fault segment location method based on the current amount and the switching capacity based on the distribution network topology in the active distribution network. Among them, the fault segment localization method based on the field device collecting information is to use the voltage and current information collected by each measuring point to obtain the electric quantity such as the similarity of the voltage and current waveform, the current amplitude ratio on both sides of the section, and the sudden change of the current phase angle are obtained to realize the positioning of the fault section. Fault section positioning based on distribution network topology is to use the distribution network topology, current and voltage measurement and various algorithms to determine the fault section.

\subsection{Fault Segment Location Based on Field Device Acquisition Information}

In [24], by analyzing the adaptability of existing distributed fault processing techniques to small current ground faults, a transient current equivalent circuit for small current ground faults suitable for distributed fault location is established. The transient zero sequence is used in [13]. On the basis of the similarity of the current waveform, the faulty section of the small current ground fault is realized by combining the effective value of the transient current of each section and the ratio of the transient zero sequence current peak on each side of the section to the power frequency zero sequence current amplitude.

Due to the randomness of the distributed power supply in the distribution network, the traditional method of identifying the fault section based on the current amplitude information is no longer applicable. By studying the current phase angle transformation before and after the fault, the current phase angle difference is proposed. Source distribution network fault section identification method [25] [26] [27]. In [25], the line fault section is judged by comparing the sudden change of the phase sequence current phase angle between the detection points on both sides of the same section. When the phase-sequence sudden change of the zero-sequence current phase angle of the detection points on both sides of the section is the same, it is determined as an out-of-zone fault; when the phase-synchronization of the zero-sequence current phase angle on both 
sides of the section is opposite, it is determined as an area fault.

In [26], the fault zone is identified by the phase angle difference of the phase currents of the detection points on both sides of each section of the distribution network. When the absolute value of the current phase angle difference in a section of the line in consecutive $\mathrm{N}$ sampling points is greater than the set threshold $\sigma$, it is determined that the section is faulty. According to the phase phase difference of the phase current of the fault, the single-phase ground short-circuit fault and the three-phase short-circuit fault can be identified. On this basis, the literature [26] defines the node zero-sequence current $\dot{I}_{0}$, and uses the node zero-sequence current to the two sections in the section. The phase failure type is further identified. If the zero-sequence current effective values at nodes $p$ and $q$ at both ends of the fault section are $I_{p}^{0}$ and $I_{q}^{0}$, respectively, the decision threshold is $I_{t h}$. When there is $I_{p}^{0}>I_{t h}$ or $I_{q}^{0}>I_{t h}$, the fault inside the section is a two-phase ground fault; Otherwise, the fault inside the zone is a two-phase phase-to-phase short circuit fault. In [27], according to the three-phase current information collected at each measuring point of the distribution network, the Clark $\alpha$ and $\beta$ mode currents are obtained by Clark transform, and then the $\alpha$ and $\beta$ mode current phase angles of each detection point are obtained by FFT transformation. The current phase angle difference between the Clarke $\alpha$ and $\beta$ modes on both sides identifies the fault section. When the absolute values of the $\alpha, \beta$ mode current phase angle difference of the detection points on both sides of a section of the low voltage active distribution network are less than the set threshold $\sigma$, it is determined that the section has not failed. When any one of the Clark's $\alpha$ and $\beta$ mode current phase angle differences in a section of the low-voltage active distribution network is greater than $\sigma$, it is determined that the section is faulty.

In [28], under the condition that the short-circuit fault current provided by the distributed power supply is lower than $70 \%$ of the short-circuit fault current provided by the system power supply, the ratio of the fault current amplitudes flowing through the upstream and downstream measuring points of each line section of the distribution network is calculated. To determine the fault zone. When $\rho \leq \gamma(\gamma \in(0.8,0.9))$, the segment is a faulty segment. However, the literature [28] is a positioning method discussed under the condition of limiting the capacity of distributed power supply to the distribution network. When the distributed power supply grid-connected conditions are relaxed, the fault segment location method is no longer reliable. In [29], under the specified DG permeability and inverter output current limit, the new longitudinal protection criterion is established by using the amplitude ratio $\rho$ of the fault current on both sides of the line and the equivalent measured impedance outside the protection line. Determine the line area/internal and external faults. The relative operating current is less than the braking current when the fault occurs outside the zone or under normal conditions. When the fault occurs in the zone, the relative operating current is greater than the braking current. 


\subsection{Fault Segment Location Based on Distribution Network Topology}

The distributed power supply is introduced into the distribution network, which makes the distribution network become a complex network with multiple power supply radiation. The switch function is constructed according to the topology of the distribution network, and different intelligent algorithms are combined to obtain the optimal fault section. Solution to achieve the positioning of the faulty section. In [30], for the problem of premature convergence of genetic algorithm, a multi-group genetic algorithm is introduced to collaborative search of group space, which avoids the algorithm falling into local optimum, and the optimal individual maintains algebra as the convergence condition, which fully improves the convergence efficiency. The simulation results show that the algorithm can locate fault segments accurately and has certain effectiveness and fault tolerance. In [31], by introducing a differential evolution algorithm into the bat algorithm, the bat algorithm lacking the mutation mechanism has the mutation, intersection and selection mechanism in the differential evolution algorithm, which improves the convergence accuracy, speed and diversity of the algorithm and avoids the population. It falls into local optimum and enhances the global optimization ability of the algorithm. In [32], the two-population evolution strategy and the information exchange mechanism are used to realize the mixture of particle swarm optimization and differential evolution algorithm. The mathematical model is solved by binary hybrid algorithm, which reduces the phenomenon of "premature convergence" in the process of fault location. The probability of improving the accuracy of fault location.

\section{Difficulties and Recommendations of Research}

According to the simulation results of the fault location method in the above distribution network literature, various positioning strategies of the faulty section of the passive distribution network can better locate the fault, but there are also many shortcomings in the signal. Extraction accuracy, related threshold settings, special fault points, and fault tolerance of the algorithm are still to be studied and improved in combination with practice.

Through the analysis of the above documents and the review of relevant data, the difficulties in fault location of passive distribution networks are: identification of fault characteristics information by field devices, applicability of fault location algorithms to complex power distribution networks, and fault information uploaded by field devices. The impact of information distortion on positioning accuracy is reflected in the weak distribution of fault characteristics of the distribution network, the interference of uncertain factors and the increasingly complex and large distribution network. Combined with the above situation, based on the existing research results, this paper believes that reference should be made to the fault line selection technology and the theoretical research of other literatures. In the collection of fault feature information, it is more ob- 
vious to collect the fault characteristics of the steady state electrical quantity through the field device. State electric quantity, obtain the characteristics of relatively prominent faults, and apply it to the research of fault location theory; for the distribution network of complex network structure, through the integration of various intelligent algorithms in the distribution network topology and characteristic electric quantity information Or matrix method, comprehensively combine the advantages of various methods and make full use of the relevant electrical quantity of the distribution network and the topology structure of the distribution network to characterize the fault characteristics, improve the fault location algorithm, solve the impact of the fault information of the fault information uploading, and improve the fault. The fault tolerance of the positioning algorithm makes fault location faster and more accurate.

However, in the fault location of the distribution network with distributed power supply, due to the integration of the distributed power supply, the traditional passive power distribution network becomes an active power distribution network, and the current direction in the distribution network is no longer unique. Moreover, the distributed power supply in the distribution network is often random, and the change of its fault characteristics brings great challenges to the coordination and cooperation of relay protection, which leads to the original distribution network fault location method is no longer applicable. Moreover, the location, capacity, and integration of different types of distributed power sources into the distribution network have different effects on distribution network fault location, which makes fault location more difficult. Combined with the existing research results, this paper believes that based on the real-time information in the distribution network, with the distribution network topology and the current direction measurement of each measurement point, combined with the advantages of various intelligent algorithms, the implementation of the distribution network site information and the main Station interactive fault real-time positioning strategy.

\section{Research Conclusions and Prospects}

From the research results of distribution network fault location, the research on fault line selection technology is the most mature. A large number of fault line selection methods have been applied to the field, but the sensitivity and reliability of its line selection are applied in actual field. In the fault section ranging, domestic and foreign scholars have carried out a lot of research, and obtained many good fault segment positioning results, and some products are applied to the site, but the fault feature information is extracted, not determining the interference of factors, special fault points and increasingly complex and large distribution networks requires a lot of theoretical and practical research; fault location is a forward-looking research, still in the theoretical research stage, the identification of measurement errors and pseudo roots. More requirements are required and more extensive and in-depth research is needed on the acquisition of algo- 
rithms and signals.

With the introduction of the national new energy strategy, distributed generation technology has developed rapidly under this strategy due to its clean, environmentally friendly and diversified and renewable energy generation technologies. When a large number of distributed power sources are integrated into the distribution network, it also brings new problems and challenges to the distribution network fault location technology. This requires researching new fault location technology for active distribution networks to achieve active distribution. The fault location of the power grid provides guarantee for the safe and reliable operation of the active distribution network.

\section{Fund}

The Project of Sichuan provincial science and Technology Department (Grant No. 2017JY0338, 2019YJ0477, 2018GZDZX0043).

The artificial intelligence key laboratory of Sichuan province Foundation (2017RYY02).

Sichuan University of Science and Engineering talent introduction project (2017RCL53).

Enterprise informatization and Internet of things measurement and control technology key laboratory project of Sichuan provincial university (2018WZY01).

The Project of Sichuan Provincial Academician (Expert) workstation of Sichuan University of Science and Engineering (2018YSGZZ04).

\section{Conflicts of Interest}

The authors declare no conflicts of interest regarding the publication of this paper.

\section{References}

[1] Guo, Z. and Wu, J. (2010) Imitative Electromagnetic Algorithm for Fault Interval Location in Distribution Network. Proceedings of the CSEE, 30, 34-40.

[2] Shu, H. (2008) Distribution Network Fault Line Selection. Mechanical Industry Press, Beijing.

[3] Ren, Q., Shu, Q. and Liu, Y. (2015) Fault Location Algorithm for Distribution Network Based on Symmetric Injection Method for Reflected Wave Feature Extraction. Power System Protection and Control, 43, 19-25.

[4] Qi, Z., Qiao, F., Huang, Z., et al. (2014) A Method for Localization of Small Current Ground Fault Based on Transient Component Genetic Algorithm. Power System Protection and Control, 42, 34-39.

[5] Ran, Y., Zhou, B., Yang, Z., et al. (2014) A Single-Ended Fault Location Method for Distribution Network with Estimation of Contralateral Information. Power System Protection and Control, 42, 25-31.

[6] Jiao, Y., Du, S., Wang, Q., et al. (2014) Distortion Information Correction Based on Information Contradiction Principle and Fault Section Location of Distribution Network. Power System Protection and Control, 42, 43-48.

[7] Liang, R., Yang, X., Xue, X., et al. (2015) Study on the Precise Localization of Sin- 
gle-Phase Ground Faults with Zero-Sequence Distribution Parameters. Transactions of China Electrotechnical Society, 30, 472-479.

[8] Jia, Q., Zheng, X., Liu, C., et al. (2017) Fault Location Method for Distribution Network Based on Fault Direction Measurement. Proceedings of the CSEE, 37, 5933-5941.

[9] Zhang, L., Xu, B., Xue, Y., et al. (2012) Transient Localization Method for Small Current Ground Fault Based on Line Voltage and Zero Mode Current. Proceedings of the CSEE, 32, 110-115.

[10] Wang, X., Tian, S., Li, Y., et al. (2012) Fault Segment Location Method for Small Current Grounding System Based on S-Transformed Characteristic Frequency Sequence. Power System Protection and Control, No. 14, 109-115.

[11] Zhang, S., Yang, J., He, Z., et al. (2015) Fault Section Location of Distribution Network Based on Line Transient Center of Gravity Frequency. Proceedings of the CSEE, 35, 2463-2470.

[12] Ma, S., Xu, B., Gao, H., et al. (2008) A Small Current Grounding Fault Location Method for Detecting Transient Zero Mode Current Correlation. Automation of Electric Power Systems, No. 7, 48-52.

[13] Xue, Y., Li, T., Li, W., et al. (2014) Transient Analysis of Small Current Ground Fault and New Method of Segment Location. Automation of Electric Power Systems, No. 23, 101-107.

[14] He, L., Shi, C., Yan, Z., et al. (2017) Fault Segment Location Method for Small Current Grounding System Based on Generalized S-Transform Energy Relative Entropy. Transactions of Electrical Engineering, 32, 274-280.

[15] Song, G., Li, G., Yu, Y., et al. (2011) Single-Phase Ground Fault Section Location Based on Phase Current Sudden Change in Distribution Network. Automation of Electric Power Systems, 35, 84-90.

[16] Chang, Z., Song, G., Huang, W., et al. (2017) Single-Phase Ground Fault Segment Location Method Based on Phase Voltage and Current Sudden Change Characteristics. Power System Technology, No. 7, 2363-2369.

[17] Ni, G., Bao, H., Zhang, L., et al. (2010) Single-Phase Fault Charging Location Criterion for Distribution Network Based on Zero Sequence Current Sudden Change. Proceedings of the CSEE, 30, 118-122.

[18] Zhang, S., He, Z., Lin, S., et al. (2013) Single-Phase Earth Fault Location Method for Resonant Grounding System Based on Transient Characteristics of Charge and Discharge. Power System Protection and Control, No. 9, 13-20.

[19] Liang, R., Jin, Z., Wang, C., et al. (2013) Fault Location of Distribution Network Based on Traveling Wave Time-Frequency Composite Analysis. Proceedings of the CSEE, 33, 130-136.

[20] Niu, R., Liang, J., Yun, Z., et al. (2015) Single-Ended Traveling Wave Ranging for Hybrid Lines Based on Fault Distance Interval. Power System Technology, 39, 156-163.

[21] Yu, Q., Yu, S. and Li, X. (2012) Adaptive Network Protection Scheme for Distributed Network. Power System Protection and Control, 40, 110-115.

[22] Wei, G., Wu, W., Hu, D., et al. (2007) The Influence of Distributed Power Supply and Its Grid on the Grid. High Voltage Engineering, No. 1, 36-40.

[23] Shen, X. and Cao, M. (2015) Research on the Influence of Distributed Power Grid Connection on Distribution Network. Journal of China Electrotechnical Society, 30, 346-351. 
[24] Song, Y., Li, T., Xue, Y., et al. (2018) Distributed Small Current Ground Fault Location Method Based on Distribution Automation System. Electric Power Automation Equipment, 38, 102-109.

[25] Si, X., Chen, Q., Gao, Z., et al. (2014) Active Distribution Network Protection Based on Current Phase Angle Mutation Direction. Automation of Electric Power Systems, 38, 97-103.

[26] Niu, G., Zhou, L., Pei, W., et al. (2017) On-Line Fault Interval Location and Identification Method for Low Voltage Active Distribution Network. Proceedings of the CSEE, 37, 2525-2538.

[27] Niu, G., Zhou, L., Pei, W., et al. (2015) Fault Location Method of Low Voltage Active Distribution Network Based on Clark Current Phase Angle Difference. Proceedings of the CSEE, 35, 15-24.

[28] Gao, M., Xu, B. and Zhang, X. (2015) Fault Location Method for Active Distribution Network Based on Fault Current Amplitude Comparison. Electric Power Automation Equipment, 35, 21-25.

[29] Xu, C. and Lu, Y. (2015) Longitudinal Protection Scheme for Distributed Power Distribution Network. Automation of Electric Power Systems, 39, 113-118, 155.

[30] Liu, P. and Li, X. (2016) Fault Segmentation Algorithm for Distribution Network with Distributed Power Supply Based on Multi-Population Genetic Algorithm. Power System Protection and Control, 44, 36-41.

[31] Luo, Q., Su, S., Liu, G., et al. (2016) Fault Segment Location of DG Distribution Network Based on Hybrid Algorithm. Electric Power System and Automation, 28, 86-90.

[32] Zhou, Q., Zheng, B., Liao, R., et al. (2013) Fault Segment Location of Distributed Power Distribution Network Based on Particle Swarm Optimization and Differential Evolution Algorithm. Power System Protection and Control, No. 4, 33-37. 